\title{
TREATMENT OF BREAST CANCER WITH DIFFERENT ANTIPROGESTINS: PRECLINICAL AND CLINICAL STUDIES
}

\author{
G. H. Bakker, B. Setyono-Han, H. Portengen, F. H. De Jong, ${ }^{\prime}$ \\ J. A. Foekens and J. G. M. KLiJN \\ Division of Endocrine Oncology (Biochemistry and Endocrinology), The Dr Daniel Den Hoed Cancer \\ Center, P.O. Box 5201, 3008 AE Rotterdam and 'Department of Endocrinology, Erasmus University, \\ Medical Faculty, The Netherlands
}

\begin{abstract}
Summary-Treatment with antiprogestins is a new treatment modality for breast cancer. Previously, in rats with DMBA-induced mammary tumors we observed significant growth inhibitory effects of chronic treatment with the antiprogestin mifepristone (RU486). In addition, in 11 postmenopausal breast cancer patients, we observed one objective response, six instances of short-term stable disease, and four instances of progressive disease. Side-effects appeared mainly due to antiglucocorticoid properties of the drug. Increased plasma estradiol levels were observed which probably resulted from ovarian (rat) and adrenal (patients) steroidogenesis.

Combined treatment with an antiestrogen in the rat model caused additive growth inhibitory effects. Tumor inhibition after single treatment with mifepristone or tamoxifen was 90 and $75 \%$, respectively. In contrast, when combined, tumor remission similar to that caused by LHRH-agonist treatment $(50 \%)$ was observed. Even higher tumor remission was found after combined treatment with mifepristone plus LHRH-agonist $(75 \%)$. In first studies in the rat model we observed significant tumor growth inhibitory effects with two new antiprogestins of seemingly greater potency which cause less unfavorable endocrine side-effects.

In conclusion: combined treatment (antiprogestin plus antiestrogen or LHRH-agonist) may be of value in endocrine threapy of breast cancer.
\end{abstract}

\section{INTRODUCTION}

Antiprogestins form a new category of antihormonal agents of potential interest in the treatment of cancer. Recently, mifepristone (RU486) became available for preclinical research and clinical testing. Besides antiprogestational properties [1-6], mifepristone has antiglucocorticoid activities as well [7-9]. Apart from application as a contraceptive agent [10-14] or as an antiglucocorticoid in the treatment of conditions related to excess corticosteroid production [9], mifepristone may be used as a growth inhibitor of several types of tumor cells.

Previously, we reported that different dosages of the progestin megestrol acetate caused tumor growth inhibition of $40-50 \%$, whereas the antiprogestin mifepristone gave rise to $80-90 \%$ inhibition $[15,16]$. This tumor inhibition was observed in spite of stimulation of the pitu-

Proceedings of the 2nd International EORTC Symposium on "Hormonal Manipulation of Cancer: Peptides, Grow'th Factors and New' (Anti-)Steroidal Agents". Rotterdam. The Netherlands, 9-11 April 1990. itary-ovarian functions, which resulted in unfavorable endocrine effects (high plasma steroid hormone and prolactin levels) [15-17]. Recently, we have also reported [18] growth inhibitory effects of chronic single treatment with mifepristone in postmenopausal patients with metastatic breast cancer. These inhibitory effects were accompanied by unfavorable sideeffects (i.e. increased plasma estradiol concentrations) due to peripheral conversion of androgens derived from hyperstimulated adrenals [18].

Therefore, the effects of combined treatment with mifepristone (to block the $\mathrm{PgR}$ ) and tamoxifen (to block the ER) or LHRH-agonists (buserelin or zoladex; to reduce estradiol secretion) were investigated.

\section{MATERIALS AND METHODS}

\section{Preclinical studies}

Hormonal agent solutions. The progestin megestrol acetate was obtained from Sigma, St Louis, MO. The antiprogestin mifepristone (RU486) was generously provided by Roussel- 
Uclaf (Dr R. Deraedt), Romainville, France. Megestrol acetate and micronized mifepristone were suspended in olive oil and administered by subcutaneous injection (final dosages, see Fig. 1). Tamoxifen-citrate was kindly donated by ICI (Pharmaceutical Division), Macclesfield, Cheshire, U.K. Tamoxifen-citrate was dissolved in physiological saline $(1 \mathrm{mg} / \mathrm{ml})$ and was administered subcutaneously (final dosage: $400 \mu \mathrm{g} / \mathrm{kg} / \mathrm{day}$ ). The LHRH-agonist buserelin was generously provided by Hoechst A.G., Frankfürt am Main, F.R.G., in a stock solution of $1 \mathrm{mg} / \mathrm{ml}$, or as a $3.3 \mathrm{mg}$ depot-preparation. For subcutaneous injections, a work solution was made by dilution of the stock solution with physiological saline (final dosage: $40 \mu \mathrm{g} / \mathrm{kg} /$ day). In addition, the LHRH-agonist zoladex was kindly provided by ICI (Pharmaceutical Division) as a $3.6 \mathrm{mg}$ implant. Control animals received daily injections of saline.

Treatment of rats. Mammary tumor induction was realized by intragastric injection of dimethylbenzanthracene (DMBA) as previously described $[15,16]$. Tumor load of each rat was determined $[15,16]$. The results of a 3 -wk treatment are expressed as relative $(\%)$ tumor load values. The relative tumor load value of a rat in a certain group is defined as the ratio of its

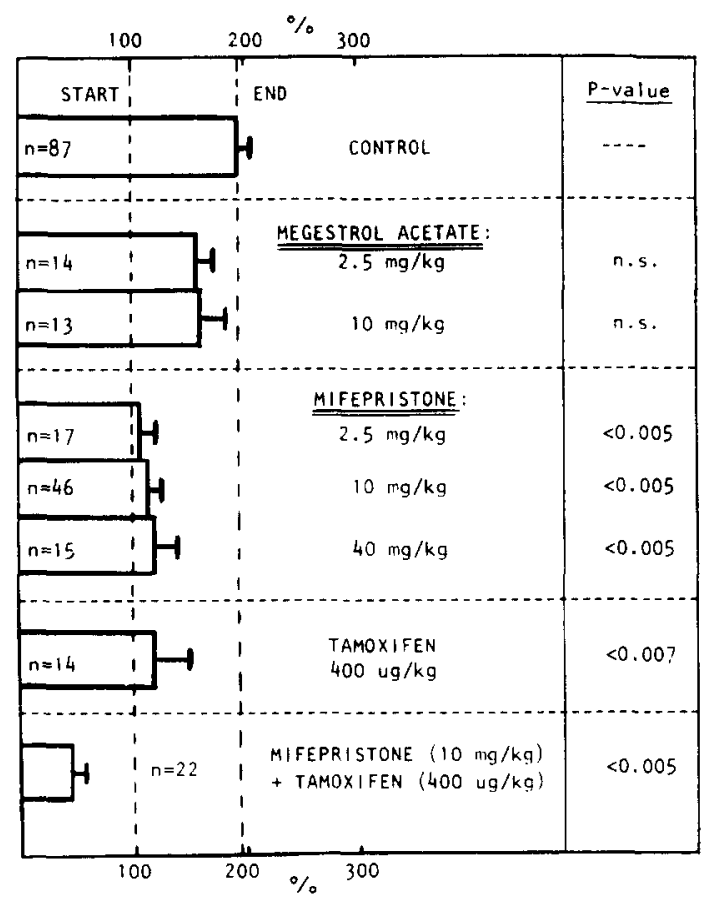

Fig. 1. Relative effects on mammary tumor load after different endocrine treatments for $3 \mathrm{wk}$. Results are means \pm SEM. The number of animals is indicated in the bars. $P$-value denotes the statistical significance vs control. Initial tumor load $=100 \%$. n.s. $=$ Not significant tumor load at the end of treatment (in $\mathrm{mm}^{2}$ ) and the average tumor load of the group as a whole (in $\mathrm{mm}^{2}$ ) at the start of treatment, and multiplied by 100 . After treatment rats were killed by decapitation, and mammary tumors, specific organs and blood were collected, as described $[15,16]$. Steroid receptor and plasma hormone assays were performed as described $[15,16]$.

Statistical evaluation of results. The significance of differences between the results obtained in the various treatment groups were calculated using Wilcoxon's test.

\section{Clinical studies}

Patients, materials and methods. This study was started after approval by a local Human Investigations Committee and by the Dutch Cancer Society (Protocol KWF-CKVO 86-09). Eleven postmenopausal patients (mean age, $63 \mathrm{yr}$; range, 46-75 yr) gave informed consent. They were treated daily during 3-34 weeks with $200-400 \mathrm{mg}$ of mifepristone (RU486, Roussel Uclaf, France) p.o. as a second-line single treatment after first-line treatment with tamoxifen, irrespective of the response to tamoxifen. In four patients, the receptor status of the primary tumor was unknown; three patients had an $\left[\mathrm{ER}^{+}, \mathrm{PgR}^{+}\right]$tumor, and four patients an $\left[\mathrm{ER}^{+}\right.$, $\mathrm{PgR}^{-}$] tumor.

Hematological, biochemical and endocrine parameters were studied in 10 patients before and after 4, 8, 12 and 16 wk of treatment (for more details, see [18]). Skin and Lymph node metastases were measured every $4 \mathrm{wk}$. X-rays of specific bone and lung lesions were performed every 3 months. Bone scans were performed before and a half year after start of treatment. Scoring of response occurred according to International Union against Cancer criteria. For statistical methods, we used a Student's paired $t$-test and the non-parametric method of Wilcoxon.

\section{RESULTS}

\section{Preclinical studies}

It was found that both LHRH-agonist implants (buserelin and zoladex) caused similar effects on all parameters studied with regard to both the direction (inhibitory or stimulatory) and magnitude of the effects. Therefore, the results with these implants were combined.

Effects of treatments on tumor growth. Treatment with the progestin megestrol acetate 
resulted in approx. 40\% tumor growth inhibition (Fig. 1). Single tamoxifen treatment and mifepristone treatment resulted in a considerable inhibition of tumor growth of approx. 75 and $90 \%$ respectively, but not in tumor remission. Combined antiprogestational and antiestrogenic treatment with mifepristone and tamoxifen gave rise to a remission of tumor growth by $50 \%$, indicating additive inhibitory effects (Fig. 1). The same tumor regression was also observed after single treatment with LHRH-agonist administered by injection (buserelin). Single LHRH-agonist treatment using implants (buserelin or zoladex) was significantly more efficacious in causing tumor remission than by treatment with daily injections of buserelin (remission by 70 vs $50 \%$, respectively, $P<0.03$; [19]). Finally, combination treatment with mifepristone and daily LHRH-agonist injections (buserelin) resulted in significantly greater tumor growth inhibitory effects than single treatment with LHRHagonist injections (approx. 75 vs $50 \%$ tumor remission, respectively, $P<0.002$; [19]). When mifepristone was combined with LHRH-agonist implants, such a combination did not result in a significantly greater antitumor effect than single treatment with LHRH-agonist implants.

Effects on mean plasma hormone concentrations. We have previously described that mifepristone treatment caused increased plasma levels of LH, estradiol and progesterone, whereas FSH levels were not significantly affected $[15,16]$. In subsequent studies FSH levels were significantly suppressed by $37 \%$ $(P<0.005 ;[19])$, while plasma concentrations of progesterone were not significantly different from those in control animals. After tamoxifen treatment all plasma hormone levels studied were decreased. Combined treatment with mifepristone and tamoxifen resulted in plasma hormone concentrations which were in between those obtained after treatment with only mifepristone or tamoxifen: estradiol and progesterone levels were unchanged, and $\mathrm{LH}$ levels were increased compared to control. However, this increase in plasma $\mathrm{LH}$ level was significantly less pronounced $(P<0.0035)$ than after single treatment with mifepristone [19].

LHRH-agonists were administered by subcutaneous injections or implants [19]. LHRHagonist treatment by injections resulted in increased levels of LH (30-fold) and FSH (2-3fold), whereas treatment with LHRH-analog implant caused no significant change in $\mathrm{LH}$ levels and significantly decreased FSH levels. The decreases in plasma steroid hormone levels were more pronounced after treatment with LHRH-agonist implants than after its injection $\left(\mathrm{E}_{2}, P<0.02 ; \mathrm{Pg}, P<0.005\right)$. In combination with mifepristone, the effects of treatments on plasma hormone concentrations were comparable to those caused by the LHRH-agonist.

Effects on organ weights and steroid receptor contents of the mammary tumors. For details on these effects of treatments we refer to [19]. Cytosolic tumor ER contents were decreased by all treatment modalities. Tumor $\mathrm{PgR}$ contents tended to increase after tamoxifen treatment and were significantly decreased after mifepristone treatment. Mifepristone treatment made the tumors nearly PgR-negative. Treatment with LHRH-agonist implants caused lower steroid hormone receptor contents than with daily injections of LHRH-agonist. Cytosolic tumor PgR level was virtually undetectable after combined treatment with mifepristone and injected LHRH-agonist.

Effects of new antiprogestins on tumor growth. A comparison of the antitumor effects of mifepristone with those of two new antiprogestins ORG31710 and ORG31806 is shown in Fig. 2. It was found that treatment with the same dosage of either of these three antiprogestins gave rise to a considerable inhibition of tumor growth (mifepristone, ORG31806) and even to a slight tumor remission (ORG31710). However, the differences between the antitumor effects of the antiprogestins were not statistically significant.

\section{Clinical studies}

Antitumor effects. One of 11 patients showed a partial response of her lymph node metastases ( $58 \%$ decrease of tumor size) lasting 5 months; six patients showed stable disease of 3-8 month duration; and four, progressive disease from start of treatment (Table 1). Anorexia and a slight degree of nausea ( 8 times); tiredness (6 times), not feeling well and dizzziness (4 times) and somnolence (4 times) were relatively frequent complaints (Table 1).

Side-effects generally did not occur in the first week of treatment, but sometimes after weeks or months of treatment, suggesting that certain plasma levels of mifepristone have to be reached before side-effects occur. Ten patients showed a decrease in body weight (mean, $3 \mathrm{~kg}$; range, $0.9-7.5 \mathrm{~kg}$ ), while patient 11 had an initial body 


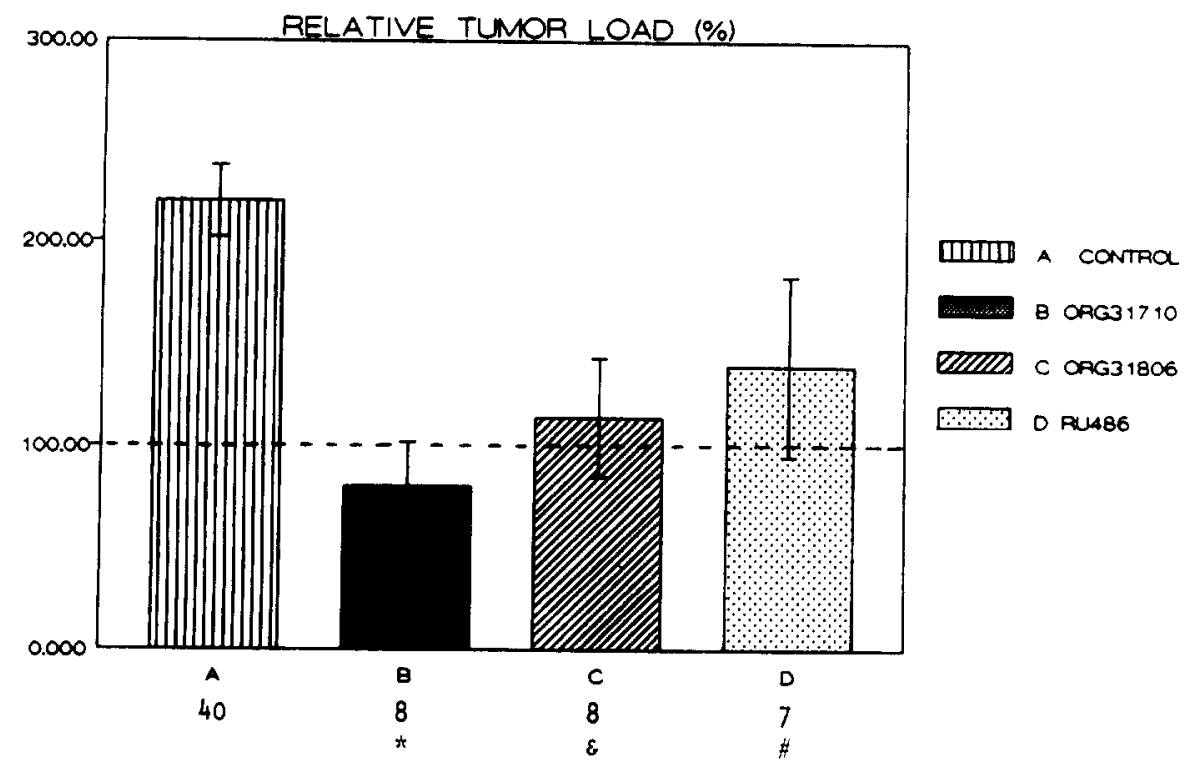

Fig. 2. Relative effects on mammary tumor load after different endocrine treatments for 3 wk with $2 \times 1 \mathrm{mg} /$ day of ORG31710, ORG31806 or RU486. Results are means \pm SEM. Below the figure are indicated the number of rats and the statistical significance vs control: ${ }^{*} P<0.05,{ }^{\&} P<0.01,{ }^{*} P<0.005$. The initial tumor load $=100 \%$.

weight increase of $5 \mathrm{~kg}$ which was followed by a decrease of $2 \mathrm{~kg}$ without other side-effects. We did not observe significant effects on blood pressure. However, one patient using antihypertensive drugs before treatment no longer required antihypertensive treatment after the start of treatment with mifepristone.

Two patients had to stop treatment with mifepristone because of side-effects (in the absence of tumor progression). One of these patients was hospitalized because of a grand mal seizure and subcoma under suspicion of cerebral metastases. Treatment with dexamethasone was instituted and mifepristone treatment was stopped. However, on the computed tomography scan of the cerebrum, metastases were not found, and the patient improved remarkably after 3 days and was dismissed from the hospital

Table 1. Antitumor and side-effects of second-line endocrine treatment with mifepristone of 11 postmenopausal patients with metastatic breast cancer

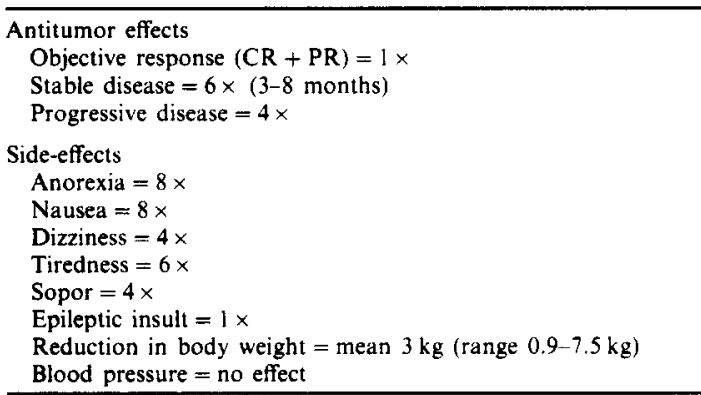

without physical complaints. These observations suggest that this complication was initiated by mifepristone. In view of the long half-life of mifepristone of about $20 \mathrm{~h}$, the improvement might be related to both excretion of mifepristone and treatment with high-dose dexamethasone that overcame the antiglucocorticoid activity of the gradually decreasing plasma levels of mifepristone.

Endocrine effects. In addition to plasma $\mathrm{ACTH}$, plasma cortisol concentrations showed a highly significant increase during treatment, indicating stimulation of the pituitary-adrenal axis [18]. In contrast to a normal increase of plasma cortisol concentrations by stimulation with synacthen before treatment, there was no significant change of the increased basal cortisol levels by synacthen during mifepristone treatment. Moreover, the increased basal cortisol concentrations could not be suppressed by $1 \mathrm{mg}$ of dexamethasone during mifepristone treatment [18]. Surprisingly, plasma androstenedione and especially plasma estradiol levels increased significantly during treatment. We found no significant effects on basal and stimulated gonadotropin concentrations, while plasma SHBG concentrations slightly decreased. Plasma estradiol concentrations were positively correlated with plasma androstenedione $(P<0.05)$ and cortisol concentrations $(P<0.01)$. Basal prolactin levels did not change 
during mifepristone treatment, while thyrotropin-releasing hormone-stimulated plasma prolactin concentrations tended to increase, possibly in relation to the increased estradiol levels.

\section{DISCUSSION}

We have previously demonstrated in experimental breast cancer studies that mifepristone is a more potent tumor growth inhibitor than the progestin megestrol acetate in rats bearing DMBA-induced mammary tumors $[15,16]$. In addition, it was found that the antitumor effect of mifepristone was not different from that of the antiestrogen tamoxifen [19]. The tumor growth inhibitory effects of mifepristone occurred in spite of increased plasma concentrations of estradiol (and prolactin; cf. [15]) as a consequence of stimulation of the pituitaryovarian axis $[15,16]$. Also, in postmenopausal women with metastatic breast cancer, chronic single second- or third-line treatment with mifepristone caused tumor growth inhibitory effects $[18,19]$. In our group of postmenopausal patients, and similar to the results in the rat, these inhibitory effects were observed in spite of increased estradiol concentrations [18]. The latter was caused presumably by stimulation of the pituitary-adrenal axis, followed by peripheral conversion of adrenal androgens into estrogens [18]. These observations, together with those of direct growth inhibition of cultured mammary tumor cells $[1-3,16]$, indicate that the main mechanism of action of antiprogestational treatment is a direct growth inhibitory effect at the level of the mammary tumor cells. The effects of combined treatment with mifepristone (to block the $\mathrm{PgR}$ ) and tamoxifen (aiming also at blockade of the ER), or with mifepristone and an LHRH-agonist (to decrease estrogen secretion) indicate that combined antiprogestational-antiestrogenic treatment with mifepristone plus tamoxifen was superior to treatment with either drug alone, i.e. combined treatment resulted in strong tumor remission compared to only tumor growth inhibition, but no remission, after treatment with either agent [19]. Previously, additive growth inhibitory effects on breast cancer cells were observed in vitro with certain concentrations of mifepristone and tamoxifen [2].

Interestingly, treatment of rats bearing DMBA-induced mammary tumors with two new antiprogestins (i.e. ORG31710 and
ORG31806) at even lower dosages than the $10 \mathrm{mg} / \mathrm{kg} /$ day previously used, resulted in a slight tumor remission. The balance of antitumor effects and unfavorable endocrine effects appeared improved on the basis of increased antitumor activities and seemingly reduced endocrine side-effects (not shown) of these new antiprogestins. The antitumor activity and the mechanism of action of other antiprogestins, such as onapristone, have been described by Schneider et al. [21] in studies of the DMBA- or MNU-induced mammary tumors in the rat.

It is concluded that in treatment with mifepristone, the additional blockade of the tumor ER by tamoxifen or the decrease in plasma steroid hormone levels caused by LHRH-agonists, prevented the unfavorable endocrine effects resulting from single treatment with mifepristone. The combined effects result in a more pronounced tumor growth inhibition during combined endocrine treatment. Moreover, new antiprogestins (and their combinations with other hormonal agents) will be tested for the appreciation of their therapeutic values in the treatment of human breast cancer.

Acknowledgements - We gratefully acknowledge receiving the hormonal agents used in this study, obtained from Roussel-Uclaf (Dr R. Deraedt, mifepristone), ICI (tamoxifen citrate, zoladex implants), Hoechst (Dr J. Sandow, buserelin solution and implants) and Organon International B.V. (ORG31710, ORG31806). In addition, we would like to thank the Dutch Cancer Society (kwf) for support (grant DDHK $\delta \mathrm{g}-6$ ).

\section{REFERENCES}

1. Bardon S., Vignon F, Chalbos D. and Rochefort H.: RU486, a progestin and glucocorticoid antagonist, inhibits the growth of breast cancer cells via the progesterone receptor. J. Clin. Endocrinol. Metab. 60 (1985) 692-697.

2. Bardon S., Vignon E., Montcourrier P. and Rochefort H.: Steroid receptor-mediated cytotoxicity of an antiestrogen and an antiprogestin in breast cancer cells. Cancer Res. 47 (1987) 1441-1448.

3. Gill P. G., Vignon F., Bardon S., Derocq D. and Rochefort H.: Difference between R5020 and the antiprogestin RU486 in anti-proliferative effects on human breast cancer cells. Breast Cancer Res. Treat. 10 (1987) $37-45$.

4. Herrmann W., Wyss R., Riondel A., Philibert D., Teutsch G., Sakiz E. and Baulieu E. E.: The effects of an antiprogesterone steroid in women: interruption of the menstrual cycle and of early pregnancy. Comptes Rendus Acad. Sci. Paris 294 (1982) 933-938

5. Horwitz K. B.: Progestins, antiprogestins and progesterone receptors in breast cancer: mechanisms and biological actions. J. Steroid Biochem. 19 (1983) 106S (abstr. 319)

6. Schaison G., George M., Lestrat N., Reinberg A. and Baulier E. E.: Effects of the antiprogesterone steroid RU486 during the mid-luteal phase in normal women. J. Clin. Endocrinol. Metab. 61 (1985) 484-489. 
7. Lamberts S. W. J., Uitterlinden P., Bons E. G. and Verleun T.: Comparison of the actions of RU38486 and megestrol acetate in the model of a transplantable adrenocorticotropin- and prolactin-secreting rat pituitary tumor. Cancer Res. 45 (1985) 1015-1019.

8. Moguilewsky M. and Philibert D.: RU38486: potent antiglucocorticoid activity correlated with strong binding to the cytosolic glucocorticoid receptor followed by an impaired activation. J. Steroid Biochem. 20 (1984) $271-276$

9. Nieman L. K., Chrousos G. P., Kellner C., Spitz I. M., Nisula B. C., Cutler G. B., Merriam G. R., Bardin C. W. and Loriaux D. L.: Successful treatment of Cushing's syndrome with the glucocorticoid antagonist RU486. J. Clin. Endocrinol. Metab. 61 (1985) 536-540.

10. Baulieu E.: Fertility control in women: results with RU486 by the end of 1985. J. Steroid Biochem. 25 (1986) $847-851$.

11. Couzinet B., Lestrat N., Ulmann A., Baulieu E. E. and Schaison G.: Termination of early pregnancy by the progesterone antagonist RU486 (mifepristone). N. Engl. J. Med. 315 (1986) 1565-1570.

12. Das C. and Catt K. J.: Antifertility actions of the progesterone antagonist RU486 include direct inhibition of placental hormone secretion. The Lancet $2, \mathrm{i}$ (1987) 599-601.

13. Ulmann A.: The antiprogestins: a recent advance in fertility regulation. J. Steroid Biochem. 27 (1987) 1009-1012.

14. Grimes D. A., Mishell Jr D. R., Shoupe D. and Lacarra M.: Early abortion with a single dose of the antiprogestin RU486. Am. J. Obstet. Gynecol. 158 (1988) $1307-1312$

15. Bakker G. H., Setyono-Han B., De Jong F. H. and Klijn J. G. M.: Mifepristone in treatment of experimental breast cancer in rats. In Hormonal Manipulation of Cancer: Peptides, Growth Factors and New (Anti)steroidal Agents (Edited by J. G. M. Klijn, R. Paridaens and J. A. Foekens), EORTC Monograph Series. Raven Press, New York, Vol. 18 (1987) pp. $39-45$

16. Bakker G. H., Setyono-Han B., Henkelman M. S., De Jong F. H., Lamberts S. W. J., Van der Schoot P. and Klijn J. G. M.: Comparison of the actions of the antiprogestin mifepristone (RU486), the progestin megestrol acetate, the LHRH-analog buserelin, and ovariectomy in treatment of rat mammary tumors. Cancer Treat. Rep. 71 (1987) 1021-1027.

17. Van der Schoot P., Bakker G. H. and Klijn J. G. M.: Effects of the progesterone antagonist RU486 (mifepristone) on ovarian activity in the rat. Endocrinology 121 (1987) 1375-1382.

18. Klijn J. G. M., De Jong F. H., Bakker G. H., Lamberts S. W. J., Rodenburg C. J. and Alexieva-Figusch J.: Antiprogestins: a new form of endocrine therapy for breast cancer. Cancer Res. 49 (1989) 2851-2856.

19. Bakker G. H., Setyono-Han B., Portengen H., De Jong F. H., Foekens J. A. and Klijn J. G. M.: Endocrine and antitumor effects of combined treatment with an antiprogestin and antiestrogen or luteinizing hormonereleasing hormone agonist in female rats bearing mammary tumors. Endocrinology 125 (1989) 1593-1598.

20. Romieu G., Maudelonde T., Ulmann A., Pujol H., Grenier J., Cavalie G., Khalaf S. and Rochefort H.: The antiprogestin RU486 in advanced breast cancer: preliminary clinical trial. Bull. Cancer 74 (1987) 455-459.

21. Schneider M. R., Michna H., Nishino Y. and El Etreby M. F.: Antitumor activity and mechanism of action of different antiprogestins in experimental breast cancer models. J. Steroid Biochem. Molec. Biol. 37 (1990) 783-787. 